\title{
Glanzmann's thrombasthenia: Working through cultural barriers
}

\author{
Colleen Tapia, Maria Tovar-Herrera
}

Glanzmann's thrombasthenia is a rare autosomal recessive bleeding syndrome characterised by a lack of platelet aggregation. This case study considers a young woman affected by this disease, integrating the role her culture plays in her medical management. Fatima (patient renamed for the purposes of this case study) is a 16-year-old girl with Glanzmann's thrombasthenia and heterozygous factor XIII deficiency, complicated by menorrhagia and a history of packed red blood cell (PRBC) transfusion for symptomatic anemia, with subsequent development of red blood cell (RBC) antibodies. Management has included years of working on hormone control, as well as dealing with the side-effects of such treatment, and starting NovoSeven (Novo Nordisk) recombinant factor VII infusions along with factor XIII replacement (Corifact; CSL Behring) via the use of a peripherally inserted central catheter (PICC), following set-backs related to hormone control. Glanzmann's thrombasthenia had its first true impact on Fatima at the onset of her menstrual cycle, just prior to the start of her teenage years. Her first menstrual cycle resulted in her admission to the intensive care unit (ICU), where emergency measures were required to save her life. When options to help Fatima began to diminish, Corifact was initiated to correct her factor XIII deficiency, thus allowing the cross-linking of fibrin to form a more stable clot. Fatima is Arab American of Palestinian descent. She has undergone HLA testing and evaluation for bone marrow transplant, however no matches have been identified to date. The family has had another child with intention of saving cord blood, but Fatima's transplant team has determined that better matches are likely to be found in unrelated donors. Pregnancy for Fatima is very high risk and believed to be life-threatening for her, which plays a very significant role in her life as a young Arab American woman.

Keywords: Glanzmann's Thrombasthenia, menorrhagia, heterozygous factor XIII deficiency, culture, bleeding

Glanzmann's thrombasthenia is a rare autosomal recessive bleeding syndrome characterised by a lack of platelet

Colleen Tapia, RN, BSN, CPN, Rush University Medical Center Hemophilia and Thrombophilia Center, Chicago, US

Email: Colleen_P_Tapia@rush.edu

Maria Tovar-Herrera, RN, BSN, Rush University Medical Center Hemophilia and Thrombophilia Center, Chicago, US. Email: Maria_Tovar-Herrera@irush.edu

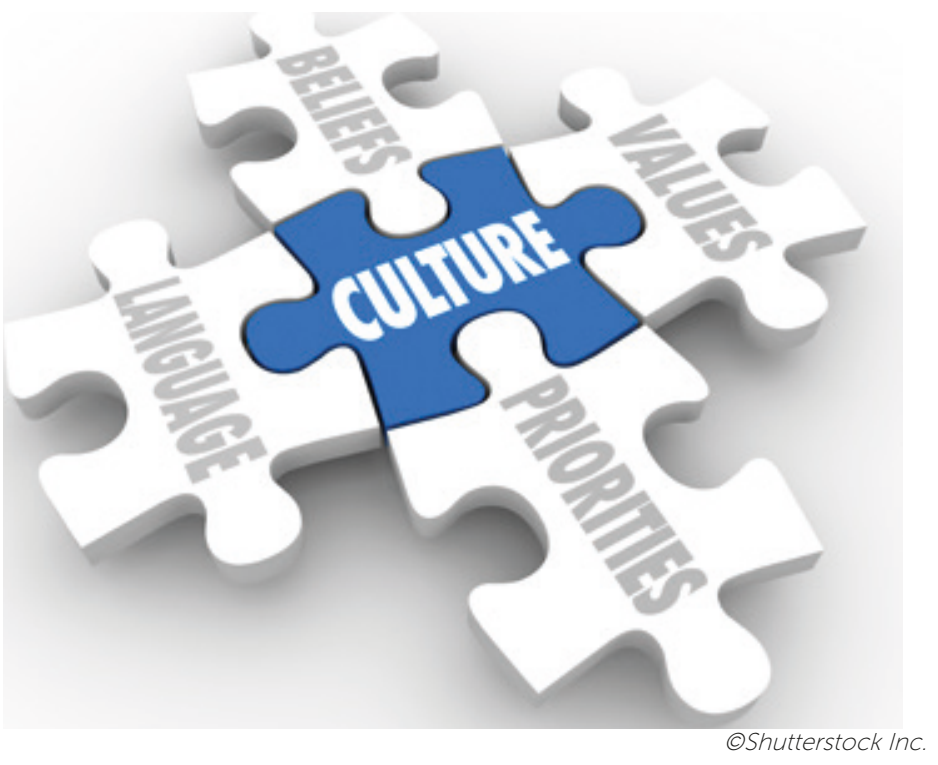

aggregation [1]. Mucocutaneous bleeding with absent platelet aggregation, in the presence of a normal platelet count is diagnostic for this condition [1]. Treatment is usually through platelet transfusion, however the severe onset of menorrhagia for women is a frequent clinical problem and is treated with high doses of progesterone [1]. Pregnancy, especially the delivery, accounts for severe haemorrhagic risk; requiring platelet transfusion prior to delivery and often continuing for at least seven days [1]. Careful consideration should be used to avoid platelet anti-HLA alloimmunisation, causing the patient to become refractory to further platelet transfusion [1].

\section{Presentation}

Fatima is a 16-year-old girl with Glanzmann's thrombasthenia and heterozygous factor XIII deficiency, complicated by menorrhagia and history of PRBC transfusion for symptomatic anemia, with subsequent development of RBC antibodies. Glanzmann's thrombasthenia had its first true impact on Fatima at the onset of her menstrual cycle, just prior to the start of her teenage years. Her first menstrual cycle resulted in her admission to the intensive care unit (ICU), where emergency measures were required to save her life. Recently (since last summer), she has experienced increased menstrual bleeding; she was admitted recently for a blood transfusion along with IV oestrogen due to uncontrolled dysfunctional uterine bleeding causing symptomatic anaemia. The bleeding improved prior to 
discharge; however, once discharged the bleeding returned along with the passing of abnormally large clots from the uterus. When options to help Fatima began to diminish, Corifact (factor XIII; CSL Behring) was initiated to correct her factor XIII deficiency, thus allowing the cross-linking of fibrin to form a more stable clot.

Fatima is Arab American of Palestinian descent. She currently resides with her parents and four siblings: a 14-year-old-brother (who also has Glanzmann's but is essentially asymptomatic), 12-year-old brother, 9-yearold sister and 4-year-old brother. Fatima has undergone HLA testing and evaluation for bone marrow transplant, however no matches have been identified to date. The family had Fatima's 4-year-old brother, with the intention of saving his cord blood for transplant; unfortunately, the cord blood was determined to not be a match. Fatima's transplant team has determined that better matches are more likely to be found in unrelated donors, but ethnicity is an issue, making this very difficult to rely on as a cure in this particular case.

The idea that pregnancy is very high risk, to the extent of being life-threatening for Fatima, is unbearable for both herself and her family to comprehend. In Arabic culture, it is common for women marry at a young age, often planning to bear children very soon afterwards in order to establish a family.

\section{Management and outcome}

Fatima comes to the outpatient clinic for infusions of NovoSeven in addition to continuing her monthly Corifact infusions, which has assisted in slowing down her menstruation. Currently, during her menses, she describes passing clots several times per day, but experiences minimal bleeding between these times and uses only two sanitary pads per day. She continues to feel fatigued and experiences occasional dizziness upon standing, but reports having no headaches and states that, overall, she feels much better. In addition to Corifact, NovoSeven infusions, and platelet infusions as needed, she now has up to four hormone pills per day and is followed closely by a gynaecologist for hormonal management. Treatment, in particular the hormone therapy, will be lifelong, as discontinuation poses a life-threatening risk of haemorrhage.

Fatima's medical management has been directly impacted by her culture on a number of occasions. For example, the family prefers that she is not seen by male physicians, especially when hospitalised, as this is generally not culturally acceptable to them. A recent occasion where cultural barriers caused problems with Fatima's medical care occurred when she went on a month-long vacation to Palestine. She travelled with Corifact, NovoSeven, and her hormone therapy in an effort to be sure her medication was immediately available. Fatima experienced a very severe case of menorrhagia while there and was hospitalized, requiring a blood transfusion for a haemoglobin level that had dropped to 5. Typically, within Arabic culture, women are not respected to have knowledge and, as a result, are frequently not heard or listened to, especially by professional Arab males [2]. Fatima's mother found that she had difficulty when attempting to explain the gravity of her daughter's situation to the Palestinian medical team, and in a desperate attempt to be heard so that Fatima's menstrual bleeding did not progress any further, she contacted the medical team who care for her in the United States. In the meantime, this delay, caused by a cultural barrier, could have put Fatima's life at risk. Luckily, the medical team in the US were able to speak directly with the medical team in Palestine and Fatima returned home safely.

\section{Discussion}

Questions surrounding this case include the care of this particular patient in a culturally sensitive way in order to maintain her compliance with the current treatment regimen. How can the medical team manage this patient should she decide to marry and want to bear children? On one occasion, Fatima stopped taking the hormone therapy due to side-effects and as a consequence, was admitted to the ICU with severe haemorrhage associated with menorrhagia. With this in mind, how will the medical team approach the family about Fatima's ability to come off of hormone therapy in order to conceive? How can her culture be respected during such conversation?

Throughout her care, the family has shared with our team that no diagnostic information is shared about Fatima within their community or among friends and/or extended family, in an effort to avoid her being perceived as not a "good catch" for a prospective husband in the future. In the absence of knowing about her severe medical condition, how will this affect critical conversations that need to occur between Fatima and her future husband should they want to conceive? The medical team caring for Fatima want her to live a life that fulfils her dreams and desires. How can this be done successfully without potentially putting her life at serious risk?

\section{Acknowledgements}

The authors wish to thank Mindy Simpson, MD and Lucy Ramirez, MSW, LCSW, for their input and review of this case study.

\section{Disclosures}

The authors have advised no interests that may be perceived as posing a conflict or bias.

This is an Open Access article distributed under the terms of the Creative Commons Attribution License (http:// creativecommons.org/licenses/by/2.0), which permits unrestricted use, distribution and reproduction in any medium, provided the original work is properly cited.

\section{References}

1. Nurden AT. Glanzmann thrombasthenia. Orphanet J Rare Dis 2006; 1: 10. doi: $10.1186 / 1750-1172-1-10$

2. Al Alhareth Y, Al Alhareth Y, Al Dighrir I. Review of women and society in Saudi Arabia. American Journal of Educational Research 2015; 3(2): 121-5. doi: 10.12691/education-3-2-3. 